\begin{abstract}
MATERNAL META-EMOTION AND CHILD SOCIO-EMOTIONAL FUNCTIONING IN IMMIGRANT INDIAN AND WHITE AMERICAN FAMILIES
\end{abstract}

\author{
by Suchi S. Daga, M.A.
}

Child emotion socialization processes vary across and within cultures, and are implicated in various child outcomes. The present study compared Indian immigrant $(\mathrm{N}=38)$ and White American $(\mathrm{N}=46)$ mothers' parental meta-emotion philosophies in the USA, specifically examining how differences in these philosophies may be related to reports of child social and behavioral outcomes. Results showed that White American mothers were rated significantly higher than Indian mothers in emotion coaching, awareness of their own and their child's emotions, and regulation of their own emotions; however the results also indicated that emotion coaching was significantly related to child outcome for White American mothers, while it was unrelated to child outcome for Indian mothers. Moderation analysis revealed that ethnicity and children's parent-oriented interdependence moderated the relationships between mothers' acceptance of their child's emotions or mothers' regulation of her own emotions and child behavioral outcomes. 


\title{
MATERNAL META-EMOTION AND CHILD SOCIO-EMOTIONAL FUNCTIONING IN IMMIGRANT INDIAN AND WHITE AMERICAN FAMILIES
}

\author{
A Thesis \\ Submitted to the \\ Faculty of Miami University \\ in partial fulfillment of \\ the requirements for the degree of \\ Master of Arts \\ Department of Psychology \\ by \\ Suchi S. Daga, M.A. \\ Miami University \\ Oxford, Ohio \\ 2012
}

Advisor

Vaishali V. Raval, Ph.D.

Reader

Jen H. Green, Ph.D.

Reader

Aaron M. Luebbe, Ph.D. 


\section{Table of Contents}

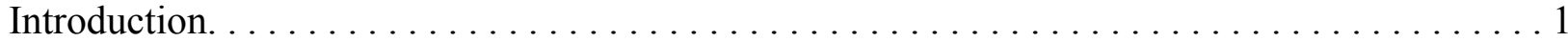

Emotion Socialization within a Socio-cultural Context. . . . . . . . . . . . . .

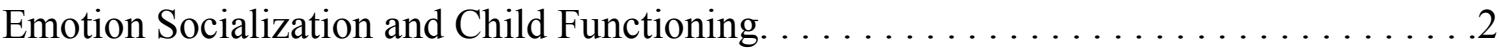

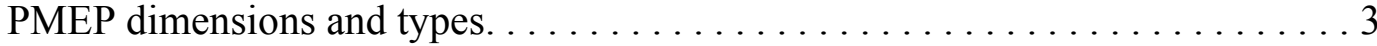

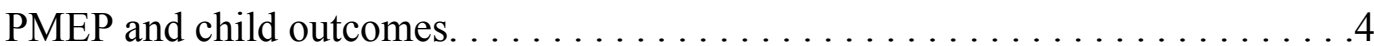

Variation in Emotion Socialization across Cultural Groups. . . . . . . . . . . . 4

The Present Study. . . . . . . . . . . . . . . . . . . . . . . 7

Method. . . . . . . . . . . . . . . . . . . . . . . . . . . . . . . . . . . .9

Participants. . . . . . . . . . . . . . . . . .

Sample demographics. ......................... 10

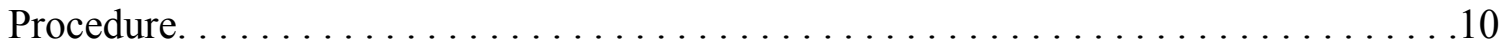

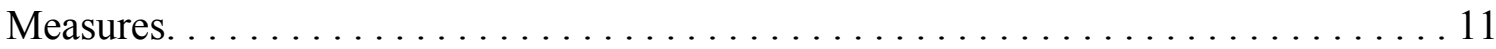

The Parental Meta-Emotion Interview. . . . . . . . . . . . . . . . 11

The Child Behavior Checklist for Ages 6 to $18 \ldots \ldots \ldots \ldots \ldots \ldots \ldots \ldots \ldots \ldots$

The Home and Community Social Behavior Scales. . . . . . . . . . . . 12

The Suinn-Lew Asian Self Identity Acculturation Scale. . . . . . . . . . . . . . 13

Parent-Oriented Interdependent Self-Construal Scale. . . . . . . . . . . . . . . 13

Results. ........................................ 13

Preliminary Analyses. . . . . . . . . . . . . . . . . . . . . . .

Research Question One: Ethnic Group Differences in PMEP. . . . . . . . . . . . . . 14

Research Question Two: Acculturation and PMEP in the Indian Immigrant Group. . . 15

Research Questions Three and Four: Moderation Analyses. . . . . . . . . . . . 16

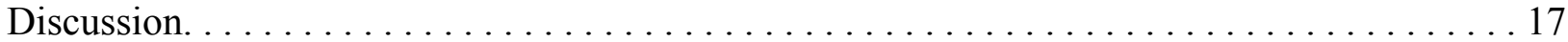

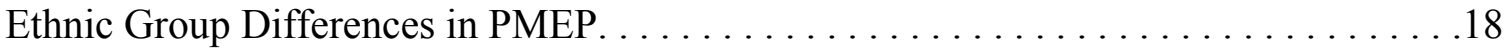

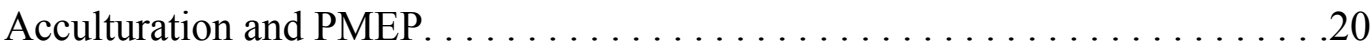

PMEP and Child Outcomes in Indian Immigrant and White American Families. . . . . .21

Limitations and Future Directions. . . . . . . . . . . . . . . . . . . . . . . 24

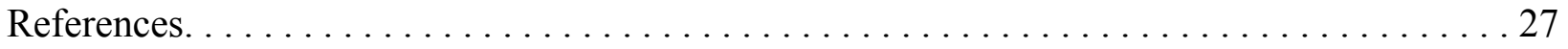




\section{List of Tables}

1. Demographic Differences among Indian Immigrant and White American Participants. .32

2. Means and Standard Deviations for Indian Immigrant and the White American

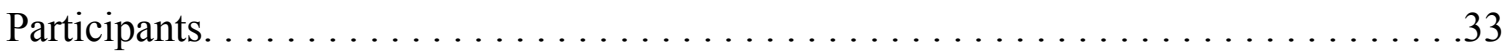

3. Intercorrelations among Indian Immigrant and White American Participants. . . . . . 34

4. Summary of Regression Analyses Testing for Ethnicity as a Moderator of the Relationship between Child Acceptance and CBCL Externalizing. . . . . . . . . . 35

5. Summary of Regression Analyses Testing for POISCS as a Moderator of the Relationship between PMEP Dimension of Child Acceptance and CBCL Internalizing. . . . . . . . 36

6. Summary of Regression Analyses Testing for Ethnicity as a Moderator of the Relationships between PMEP Dimension of Child Coaching and CBCL Externalizing and Mother-reported Home and Community Social Behavior Scales (HCSBS) . . . . . 37

7. Summary of Regression Analyses Testing for Ethnicity as a Moderator of the Relationship between Regulation and CBCL Externalizing. . . . . . . . . . . 38 


\section{List of Figures}

1. Interaction of Child Acceptance and Ethnicity (Indian or White American) in predicting CBCL Externalizing scores. . . . . . . . . . . . . . . . . . . 39

2. Interaction of Child Acceptance and Parent-oriented Interdependence (POISCS) Mean in

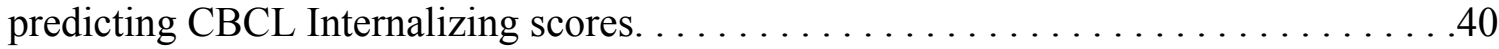

3. Interaction of Child Coaching and Ethnicity (Indian or White American) in predicting CBCL Externalizing scores. . . . . . . . . . . . . . . . . . 41

4. Interaction of Child Coaching and Ethnicity (Indian or White American) in predicting Mother-reported Home and Community Social Behavior Scales (HCSBS) . . . . . . 42

5. Interaction of Parent Regulation and Ethnicity (Indian or White American) in predicting

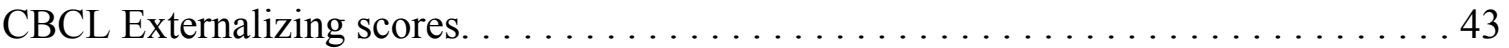


Maternal Meta-Emotion and Child Socio-Emotional Functioning in Immigrant Indian and White American Families

Within the developmental psychopathology framework, the understanding of the socialization of children's emotions has become a central issue, as emotional competence has wide-ranging implications in the development of social competence and in psychological functioning. Both social and psychological capacities, and dysfunction in these capacities, can be seen as outcomes of parental socialization of emotions, a process that includes a child's comprehension, experience, communication, and regulation of emotion (Eisenberg, Cumberland \& Spinard, 1998).

While socialization goals vary across cultures, subcultures, and other contexts (Eisenberg et al., 1998), goals are often interpreted through parenting beliefs, ethnotheories, and eventually expressed through parental behavior strategies (Keller \& Otto, 2009). Within emotion socialization literature, the construct of parental meta-emotion philosophy (PMEP) combines parents' beliefs and behaviors related to emotions. Specifically, a PMEP is defined as "an organized set of feelings and thoughts about one's own emotions and one's children's emotions" (Gottman, Katz, \& Hooven, 1996, p. 243). A growing body of literature has demonstrated that PMEPs are implicated in child outcomes in White American families in the West. However, relatively little research attention has been paid to PMEPs across cultural and ethnic groups. The present study sought to examine PMEPs in a cultural context, specifically examining the link between PMEPs and child outcomes in White American mothers and Asian Indian immigrant mothers in the United States (US).

\section{Emotion Socialization within a Socio-cultural Context}

Emotional and social competencies are based on community and cultural standards; thus, one facet of emotional socialization is the enculturation of emotion. Culture not only influences and organizes socialization goals and mechanisms (e.g., observations of emotional exchanges, opportunities for emotional experiences) in general, but also influences child-rearing practices and child development beliefs (Bornstein \& Cheah, 2006; Cole \& Tan, 2007).

While the idea that socialization occurs in a cultural context has been agreed upon by many (Bornstein \& Cheah, 2006; Bronfenbrenner, 1977), efforts to conceptualize culture have been met with various attempts. Culture may be generally defined as shared customs, practices, and beliefs that are transmitted generationally (Cole \& Tan, 2007), and Keller and Otto (2009) 
provide a conceptual framework for understanding the development of emotion regulation in cultural context. The goals parents try to accomplish through the socialization process, such as helping their child become an autonomous or socially interdependent individual, shape parents' day-to-day interactions and conversations with their children (Keller \& Otto, 2009).

In White middle-class families in Western cultures (e.g., the US), a cultural model of independence or autonomy may be salient, which conceptualizes individuals as independent, separate beings whose personal needs and desires define their sense of self and guide social behavior (Kağıtçıbaş1, 1996, 2005; Keller \& Otto, 2009; Markus \& Kitayama, 1991). Those with independent self-construals organize their behavior by referencing their own thoughts, feelings, and behaviors rather than those of others, and in social or interpersonal interactions, the focus is on the self and how to assert one's own attributes. For these individuals, emotions are experienced and expressed as internal personal characteristics, and communication of emotions symbolizes an expression of individuality (Kitayama, Mesquita, \& Karasawa, 2006). Thus, socialization practices in these families are likely to encourage the expression of emotion in children to help develop their individuality (Keller \& Otto, 2009). It is important to note, however, that although White-middle class individuals in Western cultures, like the US, are often deemed independent, variation does exist both within the US and within individuals on the independent-interdependent dimensions (Oyserman, Coon, \& Kemmelmeier, 2002; Vandello \& Cohen, 1999).

In contrast, a cultural model of relatedness (Kağıtçıbaşı, 1996, 2005) or interdependence (Markus \& Kitayama, 1991) is considered to characterize traditional communities in many nonwestern cultures as well as ethnic minority groups in the US, where individuals are conceptualized as inherently connected to others, and these social relationships define selves. Those with interdependent construals of self see their thoughts, feelings and behaviors as determined by and contingent on those of others in the relationship, and in social interactions, importance shifts to the other (Markus \& Kitayama, 1991). For individuals in these communities, emotions are experienced and expressed for the purpose of maintaining social relationships and interpersonal harmony. Thus, in non-western communities and in ethnic minority groups, socialization practices likely focus on teaching children to control negative emotions with the eventual goal of helping them become a 'socially interrelated person' who can maintain harmonious interpersonal relationships (Keller \& Otto, 2009).

\section{Emotion Socialization and Child Functioning}


The process of child emotion socialization is complex, and parental emotion-related socialized behaviors (ERSBs) influence children in numerous ways (Eisenberg et al., 1998). Eisenberg and colleagues (1998) highlighted three major ERSBs through which socialization can occur: a) parental reactions (behavioral and emotional) to children's emotions, b) parental discussion of emotions with children, and c) parental expression and modeling of their own emotions. Both child (e.g., age and gender) and parental (e.g., beliefs and values) characteristics, combined with cultural and situation-specific characteristics can all serve to impact these ERSBs. The ways in which parents react and behave toward their own and their children's emotions can then affect how a child learns and feels about regulating emotions and experiences and expresses emotions. Furthermore, the quality of the parent-child relationship, and how a child perceives the self, relationships, and the world can be impacted (Eisenberg et al., 1998). In addition to the socialization modes described by Eisenberg and colleagues' (1998), Gottman and colleagues' (1996) construct of PMEP combines several of the above mentioned aspects of emotion socialization, including parents' expression of their own emotions, parents' discussion of emotions, and parents' direct responses or management of child emotions.

PMEP dimensions and types. Based on the work with White middle-class families in the US, The four types of PMEPs identified by Gottman and colleagues (1997) are emotion coaching, emotion dismissing, emotion disapproving, and laissez-faire. The emotion coaching philosophy describes parents who are aware of emotions both in themselves and in their child, who see opportunities for teaching or relationship-building when their child is experiencing a negative emotion, who validate their child's emotions, and who help their child verbally label emotions and problem solve. Parents with an emotion dismissing PMEP, though they may be sensitive to the child's feelings and sincere in their wish to help, feel that negative emotions (e.g., anger) are unimportant and may be harmful, and thus want to change these emotions through ignoring or distracting (Gottman et al., 1996). Parents with an emotion disapproving PMEP may be aware of their child's emotions, but find negative emotions problematic and respond with criticism, punishment, and limit setting. Finally, the laissez-faire PMEP describes parents who are high in permissive acceptance of their child's emotions, but low in coaching and helping their child understand the emotional experience (Gottman et al., 1997).

These philosophies can be further broken down into seven meta-emotion dimensions, three parental dimensions and four child dimensions (Katz, Mittman, \& Hooven, 1997). The 
parental dimension of awareness (Parent Awareness) measures how aware parents are of their own emotion processes, including their abilities to distinguish emotions and their conscious experience of emotions. The parental dimension of acceptance (Parent Acceptance) focuses on how comfortable and accepting parents are of emotions, including expression and sharing of emotions. The third parental dimension of regulation (Parent Regulation) taps into difficulties parents might experience in regulating their own emotions, including intensity, frequency, and resolution of emotions.

The first child dimension, awareness (Child Awareness), gauges parental awareness of their child's emotions, including knowing which emotion the child is feeling and knowing the cause of the child's emotions. The child dimension of acceptance (Child Acceptance) assesses the parent's acceptance of the child's emotions, including empathizing with the child's emotion and how the parent talks about the child's emotion expression. The child dimension of coaching (Child Coaching) measures parental respect for the child's emotions and how the parents helps and teaches the child, including discussing the emotions and providing comfort. Finally, the fourth child dimension of regulation (Child Regulation) examines the parental account of the child's emotion regulation, including concern about the child's experience or expression and whether or not the child can self-regulate emotions.

PMEP and child outcomes. Research thus far on PMEPs has found that emotion coaching by White middle-class parents is related to soothing effects on the children's parasympathetic nervous system, greater skill in regulating negative emotions through selfsoothing, and increased ability to focus attention. Furthermore, in peer social situations, emotioncoached children have heightened awareness and ability to regulate their own emotions, and are better able to attend to the important aspects of difficult peer interactions (Gottman et al., 1996). Katz and Hunter (2007) found that mothers' emotion coaching and acceptance of their own feelings were associated with fewer adolescent depressive symptoms and externalizing problems, higher self-esteem, and better quality mother-adolescent interactions. Additionally, Ramsden and Hubbard (2002) found lower levels of mother's acceptance of children's emotion and higher levels of negative family expressiveness were associated with lower levels of children's emotion regulation, which, subsequently, was associated with children's higher levels of aggression.

\section{Variation in Emotion Socialization across Cultural Groups}


Although most meta-emotion research has been conducted with White Americans, cultural variation has been documented in other aspects of emotion socialization. For example, parents' socialization through communication can vary across cultures, with socialization in White middle-class populations focusing on emotional expression, while emotion control is often emphasized in Eastern cultures. Specifically, with regards to encouragement or discouragement of communication of emotions, White American parents often view child expression of emotions as integral for self-esteem and emotional competency development, while Non-Western parents favor emotion expression control (Cole \& Tan, 2007). Keller and Otto (2009) found that while Cameroonian Nso mothers believed that by age three, infants should learn to control their emotions, German mothers did not agree with this goal.

A second example of cultural variation in emotion socialization can be found with respect to differ parental reactions to different children's emotions across cultures. For example, Cole, Tamang and Shrestha (2006) found culturally specific emotional patterns that resulted in different emotional socialization approaches between Buddhist Tamang and Hindu Brahman children in rural Nepal. Though both groups were similar across many dimensions, (e.g., both collectivist societies), the Tamang and Brahman concepts of competent children differed. The Tamang, Tibetan Buddhists and believers in compassion and the value of self-conscious (e.g., shame) versus strong (e.g., anger) emotions, emphasized friendly and interpersonally focused children. Brahmans, Hindus and believers in their higher caste leading to spiritual purity, emphasized academic and social status. These beliefs seemed to be reflected in emotion socialization and parental response to emotion. While angry Tamang children were typically rebuked, ashamed children were usually reasoned with. Almost the opposite was found with Brahmans; angry Brahman children were reasoned with, while ashamed children were ignored.

Furthermore, Raval and Martini (2011) examined Indian mothers' emotional and behavioral responses toward children's anger, sadness and physical pain in hypothetical situations and found that the Indian mothers respond differently to physical pain than to emotions. For instance, Indian mothers felt more sympathy and less negative emotions toward pain than anger, and mothers' common behavioral response to emotions was to "make the child understand" so the child could accept and adjust to the situation, reflecting a cultural value important to becoming a socially competent person (Raval \& Martini, 2011). 
More specifically, Western research has shown that parental responses can be categorized as supportive or nonsupportive (Eisenberg et al., 1998). Supportive responses (e.g., emotionfocused or problem-focused coping), aligned with cultural models of independence, help children regulate emotion through providing comfort or support in problem solving problems. However, for cultural models of interdependence, parental responses encourage group harmony and acceptance of situation. Raval, Raval, Wehri, Wilson, and Writer (2012) examined mothers' self-reports of responses to their children's expressions of anger, sadness, and physical pain in hypothetical situations. Three groups of mothers were included, mothers in India from an oldcity working class community, mothers in India from a suburban middle-class community, and mothers in the US from a White middle-class community. Both groups of mothers from India reported more explanation-oriented and fewer solution-focused behavioral responses to their children's anger and sadness than US mothers, highlighting the need for children to understand and accept situations for better in-group relationships (Raval et al., 2012).

In both White American and Indian samples, parental supportive responses have been linked with positive outcomes, such as child social competence (Ramsden \& Hubbard, 2002; Raval et al., 2012), while nonsupportive responses (e.g., punitive/emotion minimizing behavior) have been associated with child internalizing and externalizing problems (Eisenberg, et al., 1998; Raval et al., 2011). For example, Raval and Martini (2011) found that Indian mothers of children with internalizing and externalizing problems reported more negative emotions and punitive or minimizing behaviors in response to child emotions than control-group mothers. In another study, Raval, et al. (2012) found that while mothers' solution-oriented supportive responses were not related to child outcome, mothers' explanation-oriented supportive behaviors in response to their children's anger and sadness were related to teacher-reported child adaptive functioning.

With respect to PMEPs, most of the research has been conducted in middle-class, White American populations. However, Nahm (2006) examined the relationship between PMEPs and child outcomes in White American and Korean American families. Nahm (2006) found that Korean American parents, as compared to White American parents, were less aware and less accepting of negative emotions, and engaged in less emotion coaching. The Korean American parents also showed less directive support and less positive affect in the teaching interaction. Furthermore, the decreased parental awareness and acceptance of child emotion and increased focus on the successful completion of a teaching task, may have influenced the Korean American 
children to display more tension, more focus, and less positive emotions than White American children. It is reasonable to expect then, that White American parents may be considered more aware and accepting of negative emotions and more likely to engage in emotion coaching than Asian American immigrant groups, including Asian Indian parents.

\section{The Present Study}

As the current literature suggests, parental socialization of children's emotions plays a significant role in child competency and outcome. The literature also suggests cultural variation in parental socialization of emotions, which is likely implicated in youth functioning. As a conceptualization of parental socialization, PMEPs have been linked to different child outcomes, however, only one study has examined PMEPs in a culturally diverse sample (Nahm, 2006). The present study sought to examine cultural differences in PMEPs in relation to child outcomes.

Specifically, this study explored differences between Asian Indian immigrant mothers and White American mothers with children between 8 and 16 years of age. Early adolescence to adolescence is an important developmental period to examine emotion socialization because it is a time during which many unique contextual issues arise, for example, new and more frequent social challenges with peers, greater importance of social acceptance and friendship, issues related to romantic relationships, and beginning to establish more emotional and behavioral autonomy from parents (Klimes-Dougan \& Zeman, 2007). Given such emotionally charged developmental changes in both peer and family life, the need to examine middle childhood and adolescence is great, but there is a lack of research in parental socialization during this age. Asian Indians are of particular interest as Asians have been the fastest growing race in the past decade, with Indians growing at a rate of 69.37\% (US Census, 2010). Demographically, Indians represent a well-educated and upper-middle class community in the US. For example, 63.9\% of Indians in the US have a Bachelor's degree, 59.9\% are employed in managerial or professional occupations, and the average Indian family income is over \$70,000 (US Census, 2004). Yet, little research is available to understand parental influences on child functioning in this population.

In a study that compared parental ethnotheories (cultural beliefs, goals, concerns) of Asian Indian immigrant mothers in the US with White American mothers regarding their daughters' daily activities, Raghavan, Harkness, and Super (2010) found that Indian immigrant mothers were retaining and maintaining cultural methods and ideas of parenting from their culture of origin that contrasted with White Americans. For example, behaviorally, Indian 
immigrant mothers expected obedience from their daughters and emphasized responsibility while American mothers emphasized independence for success for their daughters, aligning with the cultural self-construals of interdependence and independence, respectively. Both sets of mothers shaped their daughters' development and outcome via these parental ethnotheories.

The present study sought to add to the literature on cultural differences in emotional socialization, and specifically to differences in emotion socialization in Asian Indian immigrant and White American families. Given that the Asian Indian immigrant mothers differed from their White counterparts in their parenting practices and goals, as reflected in their ethnotheories (Raghavan et al., 2010), it seems that these differences would also be reflected in PMEPs, which may then lead to differences in child outcome. For Asian and Asian American families, a cultural model of familial interdependence is likely to be salient. Thus, we included an assessment of children's perceptions of interdependence within the parent-child relationship. Moreover, given that Indian immigrant mothers were found to retain cultural values, we expected that emotion socialization practices of mothers in India may be helpful in understanding PMEP of Indian immigrant mothers in the US. Acculturation level of Indian immigrant mothers may be associated with their PMEPs; thus, we included an assessment of acculturation in the present study. Finally, two different types of child outcomes were examined: mothers' reports of child behavior problems and mother and child's self report of child's social competence.

The following research questions were addressed in the present study:

1) Do the mothers' scores on the various PMEP dimensions vary across Indian and White American groups? Based on previous research with mothers in India (Raval \& Martini, 2011; Raval, et al., 2011) and with Asian immigrant families in the US (Nahm, 2006), it was expected, that Asian Indian immigrant mothers would score lower on the emotion coaching dimension and the parental and child acceptance dimensions than White American mothers. Previous research has demonstrated inconsistent findings concerning emotional awareness. Nahm (2006) found that Korean American mothers were less aware of their own and their children's emotions than White American mothers. In contrast, no difference was found in self-reported emotional awareness in Indian and White American college students (Snow, Becker, Raval, Raval, \& Trivedi, 2011). Thus, parent and child awareness were examined in an exploratory manner. Also, due to lack of previous research, parent and child regulation dimensions were assessed in an exploratory manner. 
2) Within the Asian Indian immigrant group, is mothers' acculturation level associated with mothers' meta-emotion philosophy? It was expected that a positive correlation would be found between mothers' acculturation level and PMEP dimensions. Specifically, the higher the mothers' acculturation level, the more likely the mother to score higher on coaching, parent and child awareness, acceptance, and regulation dimensions.

3) Does ethnicity (White American, Indian) moderate the relationship between various dimensions of PMEP and child outcomes? It was expected that for the White American group, the positive relationship between three PMEP dimensions (parental coaching, parent acceptance, child acceptance) and child social competence would be stronger than in the Asian Indian immigrant group. Moreover, for the White American group, the inverse relationship between three PMEP dimensions (parental coaching, parent acceptance, child acceptance) and child behavior problems would be stronger than in the Asian Indian immigrant group. Based on the pattern of correlations between the other PMEP dimensions (parent and child awareness, parent and child regulation) and the two child outcomes (social competence, behavior problems) in the White American and Indian immigrant groups, moderator models pertaining to these PMEP dimensions were examined in an exploratory manner.

4) Does the child's perceptions of interdependence within the parent-child relationship moderate the relationship between various dimensions of PMEP and child outcomes? It was expected that at lower levels of children's parent-oriented interdependence, the positive relationship between three PMEP dimensions (parental coaching, parent acceptance, child acceptance) and child social competence would be stronger than at higher levels of parent-oriented interdependence. Moreover, at lower levels of children's parent-oriented interdependence, the inverse relationship between three PMEP dimensions (parental coaching, parent acceptance, child acceptance) and child behavior problems would be stronger than at higher levels of parent-oriented interdependence. Based on the pattern of correlations between the other PMEP dimensions (parent and child awareness, parent and child regulation) and the two child outcomes (social competence, behavior problems) in the White American and Indian immigrant groups, moderator models pertaining to these PMEP dimensions were examined in an exploratory manner. 
Method

\section{Participants}

The participants were Asian Indian immigrant ( $\mathrm{n}=38,59.9 \%$ girls $)$ and White American ( $n=46 ; 43.5 \%$ girls) children and their mothers from Southwestern Ohio. Mothers and children were recruited through various methods, including flyers distributed to middle and high schools in three schools districts, flyers posted in Hindu and Jain temples, South Asian grocery stores, and South Asian restaurants, through community and cultural events, and through word of mouth. Interested parents contacted the researchers via email or telephone, and interviews were scheduled at the university's psychology building, a suburban branch campus of the university, or the participants' home.

Sample demographics. Several demographic differences arose between Indian immigrant mothers and White American mothers. While there was no significant difference in child age between the two groups, White American mothers were significantly older than Indian immigrant mothers $(t(79.23)=-4.02, p<.001)$ (see Table 1$)$. Regarding education, although a majority of Indian immigrant and White American mothers reported completing college degree or above, White American mothers were significantly more likely to report only completing a few years of college or less than Indian mothers $\left(\chi^{2}(1)=8.49, p<.01\right)$. While substantial proportion of White American and Indian immigrant mothers reported being employed in professional/managerial positions, Indian mothers were more likely to be homemakers or unemployed, and White American mothers were significantly more likely to be small business owners $\left(\chi^{2}(1)=5.07, p=.02\right)$. With respect to income, a majority of Indian immigrant mothers reported yearly family income of $\$ 97,200$ or more, whereas White American mothers were relatively equally distributed in income categories below $\$ 96,000$ and above $\$ 97,200\left(\chi^{2}(1)=\right.$ 7.17, $p<.01$ ) (median annual household income in Ohio is \$51, 914) (US Census Bureau, 2010). In terms of family characteristics, although a majority of White American and Indian mothers reported living in nuclear family households, Indian mothers were significantly more likely to live in a joint family $\left(\chi^{2}(2)=8.04, p=.02\right)$. Finally, the majority of Indian mothers reported their religion as Hinduism (78.4\%) and the majority of White American mothers reported their religion as Christianity or Catholicism (91.3\%).

\section{Procedure}


This study was a part of a larger project focusing on understanding the processes of emotion socialization and youth outcomes in Indian and White American families. All participant mothers and children were fluent speakers of English, and all measures were completed in English. Trained graduate students conducted mother meta-emotion interviews, and either a second graduate student or a trained undergraduate research assistant conducted the child survey. A female graduate student of Indian origin conducted meta-emotion interviews with all Indian mothers to ensure the mother's comfort and ease. Before proceeding with the interview and survey, the graduate student provided mothers with consent forms, and verbally reviewed the voluntary nature of the participation, confidentiality, and limits to confidentiality. Once the consent forms were signed and the participants feel comfortable proceeding, the interview commenced. The graduate/undergraduate research assistant met with the child in a separate room, secured verbal assent, and provided instructions for completing questionnaires. The research assistant remained available to answer any questions.

The mother meta-emotion interview was recorded on a digital audio recorder, and the child survey was completed on a laptop. Along with other measures not relevant for the present study, children completed the Parent-Oriented Interdependent Self-Construal Scale (Pomerantz, Qin, Wang \& Chen, 2009), a measure of family interdependence. Upon completion of the metaemotion interview, the mother was provided the information about an online survey to be completed within 10 days of the in-person interview. Part of the survey included demographic information questionnaire (e.g., family type, education, religion, occupation, monthly income). The Child Behavior Checklist for Ages 6 to 18 (CBCL) (Achenbach \& Rescorla, 2001) to assess child behavior problems, the Home and Community Social Behavior Scales (HCSBS) (Merrell, Streeter, Boelter, Caldarella, \& Gentry, 2001) to assess child's social ability, and an adapted version of the The Suinn-Lew Asian Self Identity Acculturation Scale (SL-ASIA) (Suinn, Ahuna, $\&$ Khoo, 1992) to assess the acculturation level of Indian mothers. Mothers received a gift card and the children received a developmentally appropriate book for their participation.

\section{Measures}

The Parental Meta-Emotion Interview. During the in-person interview, mothers completed the Parental Meta-Emotion Interview (Katz \& Gottman, 1996). This semi-structured interview assesses the mother's own experiences regarding the emotions of sadness, anger and 
fear and her philosophies on emotional control and expressiveness. Mothers are asked about their emotions, attitudes, and behavior with regards to their children's sadness, anger, and fear.

Transcribed interviews were coded on seven parent and child dimensions, across all three emotions based on an established coding system (Katz, Mittman, \& Hooven, 1997). The parent dimensions included the mother's awareness of her own sadness, anger and fear (12 items), her acceptance of her own emotions (17 items), and her regulation of her own emotions (12 items). The child dimensions included the mother's awareness of her child's sadness, anger, and fear (9 items), her acceptance of the child's emotions (13 items), her coaching of the child's emotions (11 items), and finally, the mother's account of her child's emotion regulation (9 items). Thus, all the data about PMEP dimensions, including the child dimensions, are based on mother report. Each of the seven dimensions were rated by a trained coder (SD) on a scale from "Strongly Agree" to "Strongly Disagree," with a rating for "Don't Know" as well. To establish inter-rater reliability, another trained coder (SR) coded $20 \%$ of the interviews. Bivariate correlations (Table 2) indicated adequate agreement between the ratings of both coders for all seven PMEP dimensions for Indian immigrant ( $\mathrm{r}$ values ranged from .81 to .93) and for White American mothers ( $\mathrm{r}$ values ranged from .50 to .97). These reliability estimates are comparable to previously reported reliability estimates, for example, by Katz and Nelson (2004) (r values ranged from .57 to .82) and by Nahm (2006) (r values of .70 or above).

The Child Behavior Checklist for Ages 6 to 18 (Achenbach \& Rescorla, 2001). Child behavior problems were assessed by using the Internalizing and Externalizing Scales of the CBCL. The Internalizing Scale consists of Anxious/Depressed, Withdrawn/ Depressed, and Somatic Complaints subscales and the Externalizing Scale consists of Rule-Breaking Behavior and Aggressive Behavior subscales. The responses are rated on a Likert scale ranging from Not at all true/Does not happen (0) to Very true/Happens very frequently (2). Achenbach and Rescorla (2001) reported good internal consistency (Cronbach's alpha was .90 and .94 for the Internalizing and Externalizing scales, respectively), and test-retest reliability ( $r=.91$ and .92 for Internalizing and Externalizing Scales, respectively). Internal consistency estimates in the present sample were adequate for Indian immigrant $(\alpha=.87$ and .84 for Internalizing Scale and Externalizing Scale, respectively) and White American groups ( $\alpha=.89$ and .85 for Internalizing Scale and Externalizing Scale, respectively). Though t-scores control for child age and gender, raw CBCL scores were used in analyses because CBCL t-scores were normed with White 
Americans, and the present sample consists of White American children and children of Indian descent. Thus, the original normed t-scores may not be applicable.

The Home and Community Social Behavior Scales (Merrell, Streeter, Boelter, Caldarella, \& Gentry, 2001), Mother and Child Report. As a complement to the behavioral problems assessed by the CBCL, child social competence was assessed using the 32-item HCSBA Scale A, Social Competence, which measures positive and adaptive social behaviors. The scale consists of behavioral descriptors that parents rate (e.g., "Cooperates with peers" and "Participates effectively in family or group activities") on a Likert scale from Never (1) to Frequently (5). The child participants also completed the HCSBS in a similar manner, though the questions were phrased to reflect self-ratings (e.g., "I cooperate with peers"). The HCSBS has shown high internal consistency in previous literature (Cronbach's alpha of .96 to .98 for total score). The internal consistency estimates for the present sample were consistent with those previously reported (.95 and .88 for Indian immigrant mothers and children, respectively, and .95 and .92 for White American mothers and children, respectively).

The Suinn-Lew Asian Self Identity Acculturation Scale (Suinn, Ahuna, \& Khoo, 1992). To measure the acculturation level of Indian immigrant mothers, the SL-ASIA was adapted into a 17-item questionnaire designed for participants of Indian origin. The measure includes questions about respondents' preferred languages, entertainment, food, and whom they prefer to socialize with. The questions also include the participants' perceived cultural identity and pride in cultural heritage (e.g., "If you consider yourself an Indian, how much pride do you have in this group?" and, "Rate yourself on how much you believe in American (Western values)?" Scores on the scale range from 1 to 5 , with 1 indicating preference for Asian Indian culture and customs and 5 indicating preference for mainstream American culture and customs. The SL-ASIA has shown good internal consistency in primarily East Asian American samples (Cronbach's alpha of .91), and Cronbach's alpha value of .74 in the present Indian immigrant sample was adequate.

Parent-Oriented Interdependent Self-Construal Scale (Pomerantz, Qin, Wang \& Chen, 2009). To assess how much children include their relationships with their parents in the selfconstruals, the POISCS was used. The measure consists of 16 items (e.g., "My relationship with my parents are an important part of who I am," and "I often see my parents as part of me.") that are rated on a Likert scale ranging from Not at all true of me (1) to Very true of me (5). A total score is computed that reflects parent-oriented interdependence. The measure showed good 
internal consistency in both US and Chinese samples (Cronbach's alpha of .91-.93 and .84-90, respectively), and the estimates in the present sample were somewhat lower (.68 and .77 in Indian immigrant and White American children, respectively.

\section{Results}

\section{Preliminary Analyses}

Preliminary data analyses were conducted using each PEMP dimension separately for each emotion (e.g., Parent Awareness for sadness, Parent Awareness for anger, and Parent Awareness for fear). There was no main effect of type of emotion (anger, sadness, fear) or an interaction involving type of emotion. Thus, for all further analyses, PMEP dimensions were collapsed across emotions (e.g., Parent Awareness for sadness, anger, and fear were collapsed to create a composite Parent Awareness score). All of the analyses presented here include composite PMEM dimension scores.

Due to the wide age range of participant children, the sample was divided into two groups (8-11 years old and 12-16 years old) and t-tests were conducted to determine the effect of child age on the PMEP dimensions and child outcome measures. Results showed significant effect of child age only for the PMEP dimension of Child Coaching $(t(80)=2.93, p=.004)$, with mothers rated as engaging in coaching more for younger $(M=84.22, S D=11.13)$ than older children ( $M$ $=76.17, S D=13.80)$. Preliminary analyses showed that there were no differences between Indian immigrant and White American groups in parent-oriented interdependence (the hypothesized moderator) or any of the outcome variables (see Table 2 for means).

Table 3 presents correlations among PMEP dimensions and child outcome measures separately for Indian immigrant and White American groups. For both Indian mothers and White American mothers, various PMEP dimensions (e.g., Parent Awareness and Child Awareness, Child Awareness and Child Acceptance, Child Awareness and Child Coaching, and Child Acceptance and Child Coaching) were intercorrelated. Within the White American group, parent-oriented interdependence was positively correlated with children's self reported social competence, and negatively correlated with mother reported externalizing problems. For White American mothers, the PMEP dimension of Child Coaching was negatively related to child externalizing problems, and positively related to mother-rated child social competence. In contrast, Child Coaching was unrelated to any of the outcome measures in the Indian immigrant group. For White American mothers, the PMEP dimensions of Parent Regulation and Child 
Regulation did not significantly relate with any other variable, whereas for Indian mothers, Parent Regulation was negatively related to externalizing problems, and Child Regulation positively to mother-rated child social competence. A somewhat surprising finding was that in the White American group, the PMEP dimension of Parent Awareness (parent's awareness of her own emotions) was positively correlated with her rating of child internalizing problems.

\section{Research Question One: Ethnic Group Differences in PMEP}

Mother's report of PMEP dimensions were analyzed using a 2 (ethnicity) x 2 (child gender) x 7 (PMEP dimension) multivariate analysis of variance with Child Age, Mother Age, Mother Education, Family Income, and Mother Occupation as covariates. The between subjects factors were mothers' ethnicity (Indian, White American) and child gender (male, female), and the within subjects factors were PMEP dimensions (Parent Awareness, Parent Acceptance, Parent Regulation, Child Awareness, Child Acceptance, Child Regulation, Child Coaching). Ratings on each of the PMEP dimensions were the dependent variables.

Results indicated significant main effects of ethnicity on Parent Awareness $(F(1,71)=$ $19.76, p<.001)$, Parent Regulation $(F(1,71)=4.87, p=.03)$, Child Awareness $(F(1,71)=$ $3.86, p=.05)$, and Child Coaching $(F(1,71)=7.63, p=.007)$. Results also indicated a significant main effect of child gender on Child Awareness $(F(1,71)=5.50, p=.022)$, which was qualified by a significant interaction of ethnicity and child gender $(F(1,71)=4.861, p=$ .03). Mother Education was the only covariate for which main effects were found (Parent Awareness $(F(1,71)=14.15, p<.001)$; Child Regulation $(F(1,71)=6.66, p=.01)$.

Partially consistent with the prediction, the main effect of ethnicity revealed that Indian mothers were rated as reporting less Child Coaching than White American Mothers (see Table 2), however no difference was found for Parent or Child Acceptance. Further exploratory analyses indicated that Indian mothers were also rated as reporting less Parent and Child Awareness and Parent Regulation than White American mothers. A main effect of mother's education (completing college degree or above, or some college or less) was also found for Parent Awareness and Child Regulation. Further analyses revealed no significant difference between the two groups of mothers for Parent Awareness $(t(81)=-1.83, \mathrm{p}=.07)$, but found a significant difference between the two groups of mothers for Child Regulation $(t(81)=3.66, \mathrm{p}<$ .001 ), such that mothers with less than a college degree reported higher Child Regulation than mothers with a college degree or higher. 


\section{Research Question Two: Acculturation and PMEP in the Indian Immigrant Group}

Research Question two addressed whether Indian immigrant mothers' acculturation level was related to their PMEP dimension scores. In the present sample, Indian mothers had a mean acculturation score of $2.74(S D=.37)$ on a 5-point scale where 1 indicated preference for Asian Indian culture and customs and 5 indicated preference for mainstream American culture. Individual scores ranged from 2.00 to 3.65 indicating some preference for both Asian Indian and mainstream American cultures and customs. Inconsistent with our predictions, the results indicated no significant correlations between Indian mothers' acculturation level, as measured by the SL-ASIA, and their PMEP scores (see Table 3).

\section{Research Questions Three and Four: Moderation Analyses}

Research Questions three and four addressed whether ethnicity (Indian, White American) or mean scores on parent-oriented interdependence, respectively moderated the relation between PMEP dimensions (Coaching, Parent Acceptance, Child Acceptance) and the two child outcomes (social competence as assessed by mother and child, behavior problems as reported by mother). A series of hierarchical regressions were conducted to test for moderation. Ethnicity was dummy-coded, and each of the three PMEP dimensions was centered prior to creating interaction terms. Child age was included as a covariate in all analyses in Step 1. To test whether ethnicity moderated PMEP dimensions and child outcome measures, ethnicity (dummy-coded) and each PMEP dimension (centered) were entered in Step 2 of the regression, and the two-way interaction term was entered in Step 3. The same analyses were conducted with mean scores on the measure of parent-oriented interdependence (centered) as a moderator. Additionally, for each significant interaction, a simple slope analysis was conducted to determine if the relation of the PMEP dimension to the outcome measure was significant for one or both groups.

As predicted, the ethnicity $\times$ Child Acceptance interaction significantly predicted CBCL Externalizing scores $(\beta=.30, p<.05)$ and explained additional variance in CBCL Externalizing scores $\left(\mathrm{R}^{2}\right.$ change $=.09 ; F$-change $\left.=1.91\right)$, indicating that ethnicity moderated the relationship between Child Acceptance and CBCL Externalizing scores, as shown in Table 4. Simple slope analysis revealed that the slope of the inverse relationship between Child Acceptance and CBCL Externalizing scores for White American mothers was approaching significance $(t(77)=-1.82, p$ $=.073)$, while no significant relationship was found for Indian mothers $(t(77)=1.04, p=.30)$ (see Figure 1). 
The Parent-Oriented Interdependent Self-Construal Scale (POISCS) Mean $\times$ Child Acceptance interaction significantly predicted CBCL Internalizing scores $(\beta=-.28, p<.05)$ and explained additional variance in CBLC Internalizing scores $\left(\mathrm{R}^{2}\right.$ change $=.05 ; F$-change $\left.=2.10\right)$, indicating that parent-oriented interdependence mean scores moderated the relationship between Child Acceptance and CBCL Internalizing scores, as shown in Table 5. Contrary to our expectations, however, simple slope analysis showed that the positive relationship between Child Acceptance and CBCL Internalizing scores for children with low parent-oriented interdependence scores is only significant at one standard deviation below mean parent-oriented interdependence scores $(t(74)=2.01, p=.05)$ (see Figure 2). Additionally, the negative relationship between Child Acceptance and CBCL Internalizing scores for children with high parent-oriented interdependence scores is only significant at three standard deviations above mean parent-oriented interdependence scores $(t(74)=-2.17, p=.03)$ (see Figure 3). Thus, the relationship between Child Acceptance and CBCL Internalizing Total scores is significant only at very high or at moderately low levels of parent-oriented interdependence.

Contrary to our predictions, neither parent ethnicity x Child Coaching nor parent-oriented interdependence $\mathrm{x}$ Child Coaching interactions were significant for any of the child outcomes. However, because correlations between White American mothers' Child Coaching scores and both CBCL Externalizing scores and mother-reported child social competence (HCSBS) scores were significant, these interactions were examined in more detail (see Table 6, Figures 3 and 4, respectively). With ethnicity as the moderator, simple slope analyses revealed a significant, inverse relationship between Child Coaching and CBCL Externalizing scores and a significant, positive relationship between Child Coaching and mother-reported HCSBS scores for White American mothers $(t(77)=-2.08, p=.04,(t(77)=3.01, p=.004$, respectively) and no relationship for Indian mothers $(t(77)=.18, p=.86,(t(77)=.40, p=.69$, respectively $)$.

Based on the pattern of correlations between the other PMEP dimensions (Parent Regulation, Child Regulation, Parent Awareness, Child Awareness) and child outcomes across Indian immigrant and White American groups, a few additional moderation models were tested. These exploratory analyses showed that the ethnicity $\times$ Parent Regulation interaction significantly predicted CBCL Externalizing scores $(\beta=-.47, p<.05)$ and explained additional variance in CBCL Externalizing scores $\left(\mathrm{R}^{2}\right.$ change $=.14 ; F$-change $\left.=3.19\right)$, indicating that ethnicity moderated the relationship between Parent Regulation and CBCL Externalizing scores, 
as shown in Table 7. Simple slope analysis revealed no relationship between Parent Regulation and CBCL Externalizing scores for White American mothers $(t(77)=1.04, p=.30)$, and a significant, inverse relationship for Indian mothers $(t(77)=-2.87, p=.005)$ (see Figure 5).

\section{Discussion}

The present study sought to examine cultural differences in parental socialization of the children's emotions between White American and Indian immigrant mothers. In particular, we were interested in examining ethnic differences in PMEP dimensions, and in potential moderators of the relationships between PMEP dimensions and child outcome measures. The findings, including the intercorrelations and group differences, indicate the importance of ethnicity in understanding PMEP, and the relationship between PMEP and child functioning.

\section{Ethnic Group Differences in PMEP}

Findings indicated differences between Indian and White American mothers with respect to their PMEP dimension scores. Specifically, as predicated, Indian mothers were rated as significantly lower on the PMEP dimension of Child Coaching, which included items such as, "When child is upset, Parent talks about situation, emotion," "Parent comforts during emotion," and "Parent teaches rules for appropriate expressiveness to Child" (Katz \& Gottman, 1996). This finding is consistent with Nahm (2006), who found Korean American mothers had significantly lower levels of Child Coaching than White American mothers, and studies (Raval \& Martini, 2011; Raval, et al., 2012) in which Indian mothers from India reported fewer comforting or solution oriented responses than White American mothers in the US. Thus, these combined findings suggest that Asian and Asian American mothers may engage in less emotion coaching behavior, such as validating their child's emotions or using a child's negative emotion as a teaching opportunity, than White American mothers.

Contrary to our predictions, significant ethnic group differences were not found for Parent and Child Acceptance. However, exploratory findings indicated that Indian mothers were rated as significantly lower on PMEP dimensions of Parent and Child Awareness, and Parent Regulation than White American mothers. Low awareness of Indian immigrant mothers' own and their children's emotions is consistent with Nahm's (2006) findings that Korean American mothers were rated as less aware of their own and their children's emotions than White American mothers. The PMEP dimension of Parent Awareness included items such as, "Parent experiences this emotion," "Parent is descriptive of their experience of this emotion," and 
"Parent is aware of their own remediation process" (Katz \& Gottman, 1996). The PMEP dimension of Child Awareness included items such as, "Parent notices the child has this emotion," "Parent is descriptive of some part of the remediation process," and "Parent knows cause of Child's experiences" (Katz \& Gottman, 1996). Together, these findings suggest that Asian American mothers in the US are rated as displaying lower awareness of their own and their children's negative emotions than their White American counterparts. Possibly, this difference may be due to how Indian mothers perceive and experience negative emotions. Utilizing a subsample of the dataset for the present study, Fishman (2012) analyzed metaemotion interviews of Indian mothers using open-ended qualitative methods, and found several salient themes. Some themes that emerged were the inevitability and impermanence of negative emotions, and the goal of "moving on" from negative emotions. A mother high on the Parent Awareness dimension might pay close attention to her experience of negative emotions, focusing on her physical sensations, cognitive processes, cause of emotion, and remediation process. In contrast, Fishman (2012) described Indian mothers as focusing less on the experience of their negative emotions as a way of coping with them. For example, Fishman (2012) described one Indian mother who "discusses her philosophy that paying attention to her sadness can potentially increase it, and thus how her strategy of focusing on positive things and going on to the next thing is designed to reduce her experience of sadness. It is important to note that her style of coping does not emphasize the importance of the negative emotion itself, but rather, in reducing its occurrence in her life" (p. 45). Thus, Indian mothers may demonstrate relatively lower awareness of their own and their children's emotional experiences, physical sensations, and cognitive processes as a way to deemphasize negative emotions and emphasize "moving on."

Additionally, exploratory findings showed that Indian mothers were rated lower in overall regulation of their own emotion than White American mothers. This finding is somewhat counterintuitive, particularly in light of previous findings that have indicated that Asians in general are more likely to report controlling negative emotions than White Americans (Keller \& Otto, 2009; Kuppersbusch et al., 1999; Matsumoto et al., 2008; Wilson et al., 2012). The PMEP dimension of emotion regulation included aspects such as how often and how intensely the emotion occurs, remediation techniques for the emotion, and avoidance of the emotion. Thus, this dimension does not focus exclusively on how likely is one to control felt emotion but includes the mother's ability to use various strategies to regulate emotion, and Indian mothers in 
the present study did not report frequent use of regulation strategies. Perhaps Indian mothers use different practices to regulate their emotions than those included in meta-emotion coding system. In fact, Fishman's (2012) qualitative analysis of Indian mothers' meta-emotion interviews revealed that Indian mothers reported "moving on" and use of practices such as meditation that might not have been captured by the original coding system.

A main effect of mother's education was also found for Child Regulation, such that mothers with a few years of college or less education were rated higher on emotion regulation for their children than mothers with completed college degree or higher level of education. The level of education also significantly differed across the two ethnic groups such that White American mothers more commonly reported few years of college or less education, and Indian immigrant mothers more commonly reported having completed college degree or higher education. Given this, it is likely that the impact of education on PMEP dimension of Child Regulation may simply reflect an ethnic group difference. In fact, the main effect of ethnicity on PMEP dimension of Child Regulation revealed that White American mothers had higher ratings than Indian mothers.

The PMEP differences between Indian immigrant and White American mothers might be broadly conceptualized in the context of cultural models of interdependence and independence, respectively. High scores on PMEP dimensions of awareness and coaching make sense within the cultural model of independence or autonomy that is considered salient for White middle-class families in the US (Kağıtçıbaşı, 1996, 2005; Keller \& Otto, 2009; Markus \& Kitayama, 1991), given that a focus on emotional experience and encouragement of emotional expression may be markers of individuality (Keller \& Otto, 2009). In contrast, relatively low scores on PMEP dimensions of awareness and coaching may make sense within the cultural model of interdependence or relatedness that is considered to be central to ethnic minority groups in the US (Kağıtçıbaşı, 1996, 2005; Keller \& Otto, 2009; Markus \& Kitayama, 1991). For individuals in these groups, emotions such as anger or sadness may not be readily expressed in the family context because awareness and expression may convey a discomfort with the social world that is harmful to one's relations with others in the family, and in turn, to one's sense of self (Markus \& Kitayama, 1991).

It is important to note, however, that though the mean differences of the PMEP dimensions for Indian immigrant and White American mothers are statistically significantly 
different from each other, they may not be clinically or practically different. The means of both groups are relatively close, and we must consider, for example, if the mean score of 98.84 for Indian immigrant mothers' Child Awareness and the mean score of 100.96 for White American mothers' Child Awareness are functionally different.

Acculturation and PMEP. Interestingly, for Indian immigrant mothers, acculturation scores were not correlated with PMEP dimension scores, inconsistent with our prediction that higher levels of reported acculturation would be related to higher scores on the PMEP dimensions. Indian mothers scored, on average, a $2.74(S D=.37)$ on the SL-ASIA. The low standard deviation indicates little variability among Indian mothers, indicating homogeneity with respect to acculturation. Thus, significant effects might not be evident due to restricted variability. It may also be that aspects of acculturation measured by the SL-ASIA (e.g., life style preferences such as movies, music, food, language preferences) may not impact beliefs and practices related to psychological phenomena such as emotions. Changes in one's lifestyle preferences as a result of migration to a new country may have little to do with possible changes in one's child-rearing beliefs or beliefs about emotions. Anecdotal evidence suggests that during the meta-emotion interview, many Indian mothers discussed having to adjust to or learn new practices in the US, but also emphasized maintaining traditional aspects of their culture in the US regardless of acculturation. Similarly, Raghavan and colleagues (2010) found that Indian immigrant mothers retained and maintained culturally influenced parenting methods from their original culture that differed from White Americans. Future research on parental socialization in ethnic minority families may consider utilizing measures of acculturation that tap into values and beliefs that are relevant to child-rearing.

\section{PMEP and Child Outcomes in Indian Immigrant and White American Families}

Consistent with previous research, present findings indicated that PMEP dimensions were related to child outcomes. Specifically, correlational analyses demonstrated that, for White American mothers, higher ratings of emotion coaching were related to positive child behavioral and social outcomes. In contrast, for Indian mothers, emotion coaching was not related to any child outcome. Moreover, for Indian mothers, higher regulation of their own emotions and perception of higher regulation of their children's emotions was related to positive child behavioral and social outcomes. In contrast, for White American mothers, parent or child emotion regulation was not related to any child outcome. Overall, these findings are consistent 
with previous studies have found relationships between PMEPs and physiological (Gottman et al., 1996), social (Gottman et al., 1996), and psychological (Katz and Hunter, 2007; Ramsden and Hubbard, 2002) child outcomes in primarily White middle-class samples.

In the present study, we further expected that the relationships between certain PMEP dimensions and child outcome measures would be moderated by ethnicity or parent-oriented interdependence. Consistent with our prediction, the interaction between ethnicity and PMEP dimension of Child Acceptance significantly predicted externalizing problems as assessed by mother report on the CBCL. For White American mothers, the higher the rating on Child Acceptance, the lower the CBCL Externalizing scores, and the simple slope analysis indicated that this relationship approached significance. However, the relation of Child Acceptance ratings to CBCL Externalizing scores was not significant for Indian mothers. Thus, this finding may indicate that, for White American families, acceptance of a child's negative emotions is important, as it is related to fewer maladaptive behaviors in children, however, caution needs to be exercised as the finding only approached significance.

As expected, the interaction between Parent-oriented interdependence and Child Acceptance also significantly predicted CBCL Internalizing scores, however the relationships that the interaction revealed were contrary to our expectations. High levels of parent-oriented interdependence and Child Acceptance seemed to work as protective factors, as these children had the lowest CBCL Internalizing scores, indicating that when children who are more interdependent with their parents receive more acceptance of their emotions, they display fewer internalizing behavior problems. The opposite pattern seemed to appear for children with moderately to very low parent-oriented interdependence scores, such that for children with moderately to very low levels of parent-oriented interdependence, high levels of Child Acceptance seemed related to higher levels of CBCL Internalizing scores. This finding was somewhat surprising.

In examining the Child Acceptance dimension, many of the items relate to mothers' desires to be actively involved in their child's emotional experience (e.g., "Parent want Child to talk to them about emotion," "Parent ever distracts from emotion," and "Parent ever offers treat to distract from emotion”) (Katz \& Gottman, 1996). It may be that children with low levels of interdependence may view these behaviors as being overinvolved. As Hudson and Rapee (2001) note, the parent-child relationship is an important factor for the development of anxiety disorders 
in children, and specify that previous models (Chorpita \& Barlow, 1998; Manassis \& Bradley, 1994; Rubin \& Mills, 1991) have suggested that anxious children often have parent-child relationships characterized by overprotective or overinvolved interactions. Additionally, Messer and Beidel (1994) examined third through sixth graders and found a relationship between children with anxiety disorders and their descriptions of their families promoting less independence. Thus, in the present sample, if children with low levels of interdependence view their mothers' efforts to accept their negative emotions as over-involvement or impeding their independence, this may be related to more internalizing problems, such as anxiety. In contrast, children with high levels of interdependence with their parents may view the parent-child relationship as an important part of their self-identity or see their parents as part of themselves, so that high emotional acceptance from mothers may translate to children accepting their own emotions and fewer internalizing problems.

Contrary to our predictions, the interaction between Child Coaching and ethnicity or parent-oriented interdependence did not significantly predict the child outcome measures. However, simple slope analysis revealed that for White American mothers, the higher the scores on Child Coaching, the lower the CBCL Externalizing scores and the higher the mother-reported HCSBS scores. This analysis, in combination with the significant correlations between Child Coaching and CBCL Externalizing and mother-reported HCSBS scores, and the main effect of ethnicity on Child Coaching suggest that, for White American families, emotion coaching for negative emotions is important as it is related to both few maladaptive behaviors in their children and higher child social competence.

These findings are consistent with previous literature on PMEPs in White American families that have also emphasized the importance of emotion coaching and acceptance of emotion for positive child outcomes (Katz, Maliken, \& Stettler, 2012). For example, in a recent study, White American children who received more emotion coaching regarding anger displayed fewer externalizing behavioral problems and were able to better self-regulate (Shortt, Stoolmiller, Smith-Shine, Eddy, \& Sheeber, 2010). Katz and Hunter (2007) examined adolescents with depressive symptoms and found that when mothers were more accepting and expressive of their own emotions, their children displayed fewer depressive symptoms, internalizing problems, externalizing problems, and had higher self-esteem. Improved social outcomes have also been found for children who are emotion coached by parents, for example, 
children who were provided with scaffolding, guiding language, and positive reinforcement of emotional displays were perceived to be more socially competent than children who were not emotion coached (Denham, Mitchell-Copeland, Strandberg, Auerbach, \& Blair, 1997).

Finally, exploratory analysis showed that ethnicity moderated the relationship between Parent Regulation and CBCL Externalizing scores, so that, for Indian mothers, the higher the rating on Parent Regulation, the lower the CBCL Externalizing scores. In contrast, for White American mothers, their level of Parent Regulation was not significantly related to their child's CBCL Externalizing scores. Thus, this may indicate that, for Indian families, a mother's own higher levels of emotion regulation is related to fewer displays of maladaptive emotional expression in her child. It may be that Indian mothers who are able to regulate their emotions are able to teach their children, directly or through modeling, how to regulate their own emotions and control emotional expression. Previous literature does indicate that Asian and Asian immigrant groups focus on socialization practices that encourage control of negative emotions in order to help children harmoniously maintain interpersonal relationships (Fishman, 2012; Keller \& Otto, 2009; Raval \& Martini, 2011).

It is important to acknowledge that the relationship between parenting and child outcome is reciprocal. While it may be that parental socialization practices impact child outcome, it is also possible for child behavior or temperament to affect or elicit specific parenting behaviors (Bell, 1968; 1979; Putnam, Sanson, \& Rothbart, 2002). For example, Huh, Tristan, Wade, and Stice (2006) examined the relationship between adolescent females and their parents, and found that child behavior problems were more consistent in predicting parenting than parenting was of child behavior problems. Thus, the present findings do not indicate causal relationships between PMEP dimensions and child outcome measures, and instead it is encouraged to consider how bidirectional relationships may be possible.

\section{Limitations and Future Directions}

The findings of this study present interesting and meaningful insights of child emotion socialization practices in Indian immigrant and White American families; however, it is also important to note some limitations. Although significant efforts were made to recruit families to participate in this study, the total number of participant mothers in the present study was relatively low, likely impacting the power of analyses that were conducted, specifically tests of moderation. Future examinations of PMEPs in ethnically diverse groups may use larger sample 
sizes to address this potential difficulty. Additionally, the present study only used mother report of PMEP dimensions and child behavior problems. Future studies may incorporate multiple informants, such as fathers, or additional data collection methods, such as observations of parentchild interaction. A further limitation to the present study may be challenges related to the application of the original meta-emotion coding scheme for use with Asian Indian mothers. Fishman (2012) demonstrated, through his qualitative analysis of meta-emotion interviews, that themes particular to Indian mothers were not fully captured by the original coding scheme, thus likely impacting their scores and portrayal on the PMEP dimensions. Similarly, it is important to consider appropriateness of the child outcome measures for the immigrant Indian families. For example, though the CBCL has been standardized for the US population that includes representative minority groups, as well as an Indian language translation of the CBCL is standardized with mothers living in India. Moreover, the CBCL scales in the present sample showed adequate reliability in both White American and Indian groups. However, it is important to acknowledge that the CBCL may not have captured culturally salient or unique aspects of problem behavior in the Indian children. Finally, it is also important to note that both Indian immigrant and White American communities in the US may differ based on region and sociodemographics. Our sample was primarily middle and upper-middle class, recruited from the Midwest, and caution is suggested in generalizing the present findings to all White American or Indian immigrant families in the US. Future emotion socialization studies comparing diverse Indian immigrant and White American communities in the US are encouraged. Based on the present findings, and those of Nahm (2006), future research might examine additional minority groups in the US to determine what parental emotion socialization practices positively influence child outcome. It may also be beneficial to examine ethnic minority groups with greater variability in acculturation level to determine if acculturation impacts socialization practices and child outcome. For example, future research might examine whether greater acculturation leads to more emotion coaching by minority parents, and whether this produces child outcome results similar to White American children.

Despite these limitations, the present study makes contributions to the limited literature on cross-ethnic differences in parental meta-emotion socialization practices. As ethnic and cultural diversity continues to increase in the US, so does the importance of developing culturally appropriate theories of emotion socialization. The extant literature demonstrates that 
emotion socialization can impact multiple domains of child outcome, and the present study demonstrates that ethnicity can impact the emotion socialization process, and the relation between emotion socialization and child outcomes. Thus, a better understanding of cultural influences on emotion socialization may have practical implications for future child outcomes. The findings of this study may inform culturally sensitive family interventions or parenting strategies on socializing children's emotions with practices related to positive child behavioral and social outcomes. For example, practitioners working with Indian immigrant families in the US who have children displaying maladaptive externalizing problems may consult with others to determine culturally relevant parenting behaviors that may be related to adaptive child outcomes. For example, parental coaching and solution-oriented responses may not be the most relevant parenting approaches for this group. Overall, the present study highlights the importance of conducting culturally informed research and practice in understanding parenting practices and child outcomes, and in intervening with child psychopathology. 


\section{References}

Achenbach, T. M., \& Rescorla, L. A. (2001). Manual for the ASEBA School-Age Forms \& Profiles. Burlington, VT: University of Vermont, Research Center for Children, Youth, \& Families.

Bell, R. Q. (1968). A reinterpretation of the direction of effects in studies of socialization. Psychological Review, 75, 81-95.

Bell R. Q. (1979). Parent, child, and reciprocal influences. American Psychologist, 34, 821-826.

Bornstein, M. H., \& Cheah, C. S. L. (2006). The place of "culture and parenting" in an ecological contextual perspective on developmental science. In K. H. Rubin \& O. B. Chung, (Eds.). Parental beliefs, parenting, and child development in cross-cultural perspective (pp. 3-31). London, UK: Psychology Press.

Bronfenbrenner, U. (1977). Toward and experimental ecology of human development. American Psychologist, 32, 513-531.

Chorpita, B. F., \& Barlow, D. H. (1998). The development of anxiety: The role of control in the early environment. Psychological Bulletin, 124, 3-21.

Cole, P. M., \& Tan, P. Z. (2007). Emotion socialization from a cultural perspective. In J. E. Grusec \& P. D. Hastings (Eds.), Handbook of socialization: Theory and research (pp. 516-542). New York, NY: Guilford Press.

Cole, P. M., Tamang, B. L., \& Shrestha, S. (2006). Cultural variations in the socialization of young children's anger and shame. Child Development, 77(5), 1237-1251.

Denham, S. A., Mitchell-Copeland, J., Strandberg, K., Auerbach, S., \& Blair, K. (1997). Parental contributions to preschoolers' emotional competence: Direct and indirect effects. Motivation and Emotion, 21, 65-86.

Eisenberg, N., Cumberland, A., \& Spinard, T. L. (1998). Parental socialization of emotion. Psychologial Inquiry, 9(4), 241-273.

Fishman, J. L. (2012). Phenomenological cultural investigation of Indian immigrant mothers' meta-emotion philosophy. Doctoral dissertation, Miami University.

Gottman, J. M., Katz, L. F., \& Hooven, C. (1997). Meta-Emotion: How families communicate emotionally, links to child peer relations, and other developmental outcomes. Mahwah, NJ: Lawrence Erlbaum Associates. 
Gottman, J. M., Katz, L. F., \& Hooven, C. (1996). Parental meta-emotion philosophy and the emotional life of families: Theoretical models and preliminary data. Journal of Family Psychology, 10(3), 243-268.

Hudson, J. L., \& Rapee, R. M. (2001). Parent-child interactions and anxiety disorders: An observational study. Behaviour Research and Therapy, 39, 1411-1427.

Huh, D., Tristan, J., Wade, E., \& Stice, E. (2006). Does problem behavior elicit poor parenting? A prospective study of adolescent girls. Journal of Adolescent Research, 21, 185-204.

Kağıtçıbaşı, C. (1996). The autonomous-relational self: A new synthesis. European Psychologist, 1, 180-186.

Kağıtçıbaşı, C. (2005). Autonomy and relatedness in cultural context: Implications for self and family. Journal of Cross-Cultural Psychology, 36(4), 403-422.

Katz, L. F., \& Gottman, J. M. (1996). The meta-emotion interview. Unpublished manual, University of Washington, Seattle, WA.

Katz, L. F., \& Hunter, E. C. (2007). Maternal meta-emotion philosophy and adolescent depressive symptomatology. Social Development, 16(2), 343-360.

Katz, L.F., Maliken, A.C., \& Stettler, N. M. (2012). Parental meta-emotion philosophy: A review of research and theoretical framework. Child Development Perspectives, 6, 417-422.

Katz, L. F., Mittman, A., \& Hooven, C. (1997). The Meta-Emotion Coding System. Unpublished manuscript.

Keller, H., \& Otto, H. (2009). The cultural socialization of emotion regulation during infancy. Journal of Cross-Cultural Psychology, 40(6), 996-1011.

Kitayama, S., Mesquita, B., \& Karasawa, M. (2006). The emotional basis of independent and interdependent selves: Socially disengaging and engaging emotions in the US and Japan. Journal of Personality and Social Psychology, 91, 890-903.

Klimes-Dougan, B., \& Zeman, J. (2007). Introduction to the special issue of social development: Emotion socialization in childhood and adolescence. Social Development, 16, 203-209.

Kupperbusch, C., Matsumoto, D., Kooken, K., Loewinger, S., Uchida, H., Wilson-Cohn, C., \& Yrizarry, N. (1999). Cultural influences on nonverbal expressions of emotion. In P. Philippot, R. S. Feldman, \& E. J. Coats (Eds.), The social context of nonverbal behaviour (pp. 17-44). Cambridge, UK: Cambridge University Press.

Manassis, K., \& Bradley, S. J. (1994). The development of childhood anxiety disorders: Toward 
an integrated model. Journal of Applied Developmental Psychology, 15, 345-366.

Markus, H. R., \& Kitayama, S. (1991). Culture and the self: Implications for cognition, emotion, and motivation. Psychological Review, 98, 224-253.

Matsumoto, D., Yoo, S. H., Nakaagawa, S., Alexandre, J., Altarriba, J., Anguas-Wong, A. M.,...Zengeya. A. (2008). Culture, emotion regulation, and adjustment. Journal of Personality and Social Psychology, 96, 925-937.

Merrell, K. W., Streeter, A. S., Boelter, E. W., Caldarella, P., \& Gentry, A. (2001). Validity of the Home and Community Social Behavioral Scales: Comparisons with five behaviorrating scales. Psychology in the Schools, 38(4), 313-325.

Messer, S. C., \& Beidel, D. C. (1994). Psychosocial correlates of childhood anxiety disorders. Journal of the American Academy of Child and Adolescent Psychiatry, 33, 975-983.

Nahm, E. Y. (2006). A cross-cultural comparison of Korean American and European American parental meta-emotion philosophy and its relationship to parent-child interaction (Doctoral Dissertation). Available from Dissertations and Theses database (UMI No. 3224266).

Oyserman, D., Coon, H. M., \& Kemmelmeier, M. (2002). Rethinking individualism and collectivism: Evaluation of theoretical assumptions and meta-analyses. Psychological Bulletin, 128, 3-72.

Pomerantz, E. V., Qin, L., Wang, Q., \& Chen, H. (2009). American and Chinese early adolescents' inclusion of their relationships with their parents in their self-construals. Child Development, 80(3), 792-807.

Putnam, S. P., Sanson, A. V., \& Rothbard, M.K. (2002). Child temperament and parenting. M. H. Bornstein (Ed.), Handbook of parenting: Volume 1, children and parenting (255-278). Mahwah, NJ: Lawrence Erlbaum Associates, Inc.

Raghavan C. S., Harkness, S., \& Super, C. M. (2010). Parental ethnotheories in the context of immigration: Asian Indian immigrant and Euro-American mothers and daughters in an American town. Journal of Cross-Cultural Psychology, 41(4), 617-632,

Ramsden, S. R., \& Hubbard, J. A. (2002). Family expressiveness and parental emotion coaching: Their role in children's emotion regulation and aggression. Journal of Abnormal Child Psychology, 30(6), 657-667.

Raval, V. V., \& Martini, T. S. (2011). "Making the child understand:" Socialization of emotion in urban India. Journal of Family Psychology, 25, 847-856. 
Raval, V. V., Raval, P. H., \& Deo, N. (2012). Mothers' socialization of emotion, child emotion regulation, and child socio-emotional functioning among early adolescents in India. Revised manuscript under review. Journal of Early Adolescence.

Raval, V. V., Raval, P. H., Wehri, Wilson, and Writer (2012). Mothers' socialization of children's emotion in India and United States: A cross- and within-culture comparison. In press, Social Development.

Rubin, K. H., \& Mills, R. S. L. (1991). Conceptualizing developmental pathways to internalizing disorders in childhood. Canadian Journal of Behavioural Science, 23, 300-317.

Shortt, J. W., Stoolmiller, M., Smith-Shine, J. N., Eddy, J. M., \& Sheeber, L. (2010). Maternal emotion coaching, adolescent anger regulation, and siblings' externalizing symptoms. Journal of Child Psychology and Psychiatry, 51, 799-808.

Snow, N. L., Becker, S. P., Raval, V. V., Raval, P. H., \& Trivedi, S. S. (2011, March-April). Difficulties with Emotion Regulation and Adjustment: A Comparison of College Students in India and the United States. Poster presented at the Biennial Meeting of the Society for Research in Child Development, Montreal, QC.

Suinn, R. M., Ahuna, C., \& Khoo, G. (1992). The Suinn-Lew Asian Self-Identity Acculturation Scale: Concurrent and factorial validation. Educational and Psychological Measurement, 52, 1041-1046.

Thompson, R. A., \& Meyer, S. (2007). The socialization of emotion regulation in the family. In J. Gross (Ed.), Handbook of emotion regulation (pp. 249-268). New York: Guilford.

US Census Bureau. (2010). 2010 demographic profile data. Retrieved September 12, 2011, from http://factfinder2.census.gov/faces/tableservices/jsf/pages/productview.xhtml?pid=DEC_ 10_DP_DPDP1\&prodType=table

US Census Bureau. (2004). We the people: Asians in the United States. Retrieved September 12, 2011, from http://www.census.gov/prod/2004pubs/censr-17.pdf

US Census Bureau. (2010). Income, poverty and health insurance coverage in the United States: 2010. Retrieved July 10, 2012, from http://www.census.gov/newsroom/releases/archives/ income_wealth/cb11-157.html

Vandello, J. A., \& Cohen, D. (1999). Patterns of individualism vs. collectivism across the United States. Journal of Personality and Social Psychology, 77, 279-292.

Wilson, S., Raval, V. V., Raval, P. H., Wehri, J., \& Panchal, I. N. (2012). Emotion 
expression and control in school-age children in India and the United States. MerrillPalmer Quarterly, 58, 50-76. 
Table 1

Demographic Differences among Indian Immigrant and White American Participants

\begin{tabular}{lcc}
\hline & $\begin{array}{c}\text { Indian Immigrant } \\
(\mathrm{N}=38)\end{array}$ & $\begin{array}{c}\text { White American } \\
(\mathrm{N}=46)\end{array}$ \\
\hline Mother's age & 39.05 years $(3.09)$ & 42.41 years $(4.50)$ \\
Child's age & 11.42 years $(2.23)$ & 11.48 years $(1.71)$ \\
Child gender (\% female) & $59.5 \%$ & $43.5 \%$ \\
Mother's education & & \\
A few years of college or below & $3 \%$ & $26.1 \%$ \\
College degree or above & $97 \%$ & $73.9 \%$ \\
Mother's occupation & & \\
Homemaker/Unemployed & $52.8 \%$ & $28.3 \%$ \\
Secretarial/Clerical & $2.8 \%$ & $8.7 \%$ \\
Managerial/Professional & $44.4 \%$ & $50.0 \%$ \\
Business owner & $0 \%$ & $13.04 \%$ \\
Yearly family income & & \\
Below \$96,000 & $25 \%$ & $54.35 \%$ \\
Above \$97,200 & $75 \%$ & $45.65 \%$ \\
Mother's marital status (\% married) & $97.4 \%$ & $91.3 \%$ \\
Household & & \\
Joint & $13.2 \%$ & $0 \%$ \\
Nuclear & $81.6 \%$ & $100 \%$ \\
Religion & & \\
Hinduism & $78.4 \%$ & $01.3 \%$ \\
Christianity/Catholicism & $5.4 \%$ & \\
Average years in U.S. & 13.60 years $(4.15)$ & \\
\hline Note: Mother's age, child's age, and average years in U.S. M (SD). & \\
\end{tabular}


Table 2

Means and Standard Deviations for Indian Immigrant and the White American Participants

\begin{tabular}{lccc|ccc}
\hline & \multicolumn{3}{c}{ Indian Immigrant } & \multicolumn{3}{c}{ White American } \\
Variable & $M$ & $S D$ & $r$ & $M$ & $S D$ & $r$ \\
\hline PMEP Dimensions & & & & & & \\
\hline Parent Awareness* & 128.71 & 8.65 & .87 & 134.28 & 6.07 & .61 \\
Parent Acceptance & 121.92 & 17.47 & .97 & 128.52 & 17.06 & .89 \\
Parent Regulation* & 104.55 & 8.79 & .79 & 107.39 & 5.26 & .50 \\
Child Awareness* & 98.84 & 9.30 & .93 & 100.96 & 5.73 & .73 \\
Child Acceptance & 56.69 & 8.92 & .81 & 59.28 & 8.64 & .70 \\
Child Coaching* & 75.79 & 13.83 & .89 & 83.89 & 11.41 & .97 \\
Child Regulation & 71.71 & 7.30 & .85 & 74.74 & 7.11 & .88 \\
\hline Moderator/ Outcomes & & & & & & \\
\hline POISCS Mean & 3.60 & .60 & -- & 3.47 & .68 & -- \\
SL-ASIA Mean & 2.74 & .37 & -- & N/A & N/A & -- \\
CBCL Internalizing & 36.46 & 5.81 & -- & 37.91 & 7.04 & -- \\
CBCL Externalizing & 31.05 & 3.91 & -- & 31.98 & 4.68 & -- \\
HCSBS Mother & 136.86 & 15.13 & -- & 132.00 & 17.26 & -- \\
HCSBS Child & 129.64 & 25.11 & -- & 128.67 & 13.98 & -- \\
\hline Note: The mens & & & & & & \\
\hline
\end{tabular}

Note: The means reported ehre are actual means.

* indicates significant main effect of ethnicity on these dimensions.

Ethnic group differences in PMEP dimensions was tested using MANOVA; ethnic group differences in the moderator and outcome variables were assessed using independent samples t-tests. 


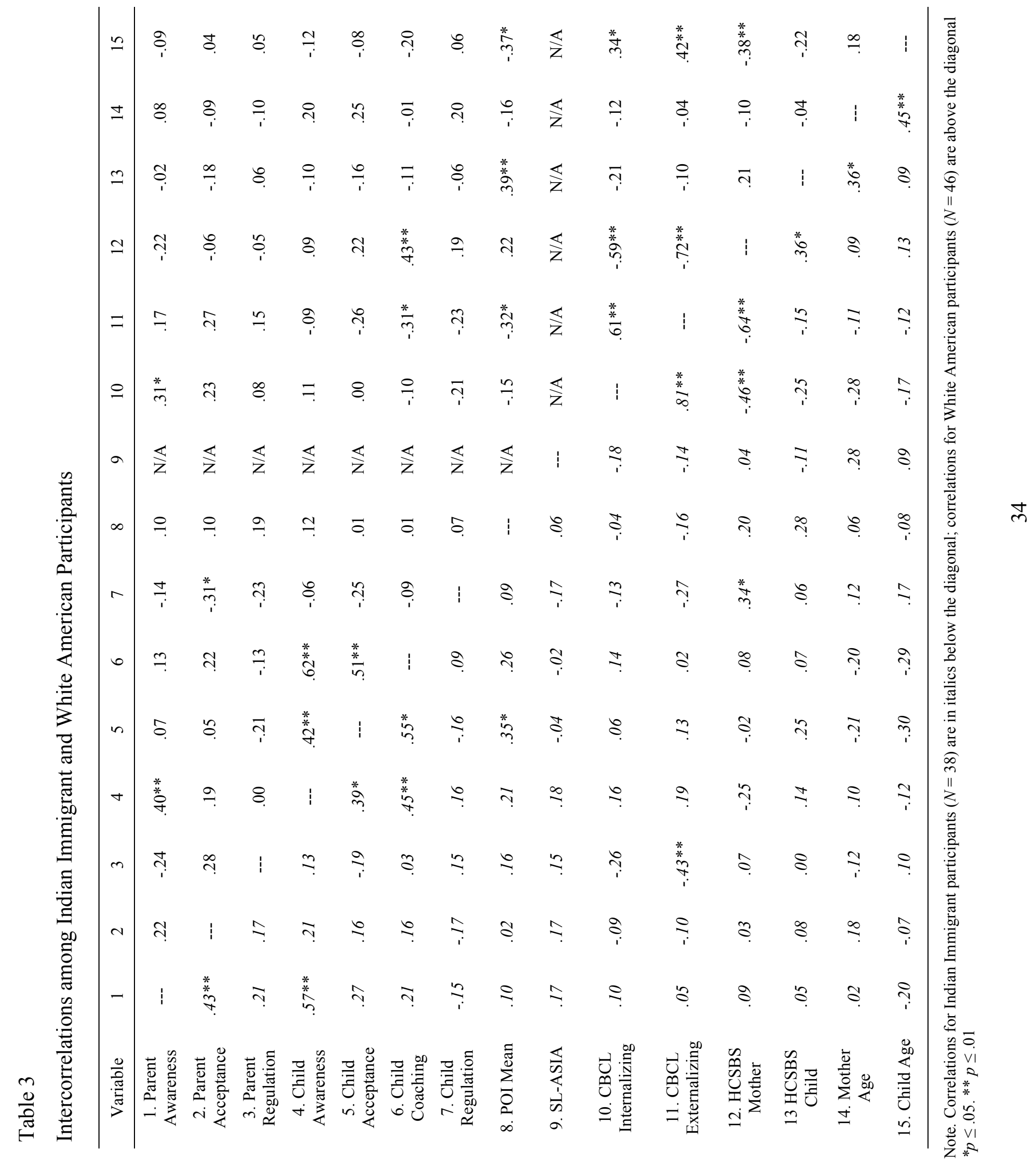


Table 4

Summary of Regression Analyses Testing for Ethnicity as a Moderator of the Relationship between Child Acceptance and CBCL Externalizing.

\begin{tabular}{|c|c|c|c|c|c|}
\hline & \multicolumn{5}{|c|}{ CBCL Externalizing } \\
\hline & $R^{2}$ & $\underset{2}{\Delta R}$ & $\Delta F$ & $\beta$ & $t$ \\
\hline Step 1 Model Summary & .02 & .03 & 2.45 & & \\
\hline Child Age & & & & .17 & 1.56 \\
\hline Step 2 Model Summary & .01 & .04 & 1.17 & & \\
\hline Ethnicity $^{\mathrm{a}}$ & & & & -.10 & -.90 \\
\hline Child Acceptance & & & & -.08 & -.66 \\
\hline Step 3 Model Summary & .04 & .09 & 1.91 & & \\
\hline Ethnicity $\times$ Child Acceptance & & & & .30 & $2.00 *$ \\
\hline
\end{tabular}

Note. Child Acceptance is centered.

$* p<.05$.

${ }^{a}$ Ethnicity dummy coded $(1=$ Indian, $0=$ American $)$. 
Table 5

Summary of Regression Analyses Testing for POISCS as a Moderator of the Relationship between PMEP Dimension of Child Acceptance and CBCL Internalizing.

\begin{tabular}{lccccc}
\hline & \multicolumn{5}{c}{ CBCL Internalizing } \\
\cline { 2 - 6 } & $R^{2}$ & $\Delta R^{2}$ & $\Delta F$ & $\beta$ & $t$ \\
\hline Step 1 Model Summary & .00 & .01 & .85 & & .10 \\
$\quad$ Child Age & & & & .92 & \\
Step 2 Model Summary & -.02 & .02 & .61 & & -.89 \\
$\quad$ POISCS & & & & -.11 & .52 \\
$\quad$ Child Acceptance & & & & .06 & \\
Step 3 Model Summary & .10 & .05 & 2.10 & & -.28 \\
$\quad$ POISCS $\times$ Child Acceptance & & & & $-2.53^{*}$ \\
\hline
\end{tabular}

Note. POISCS mean and Child Acceptance are centered. $* p<.05$. 
Table 6

Summary of Regression Analyses Testing for Ethnicity as a Moderator of the Relationships between PMEP Dimension of Child Coaching and CBCL Externalizing and Mother-reported Home and Community Social Behavior Scales (HCSBS).

\begin{tabular}{|c|c|c|c|c|c|c|c|c|c|c|}
\hline & \multicolumn{5}{|c|}{ CBCL Externalizing } & \multicolumn{5}{|c|}{ Mother HCSBS } \\
\hline & $R^{2}$ & $\Delta R^{2}$ & $\Delta F$ & $\beta$ & $t$ & $R^{2}$ & $\Delta R^{2}$ & $\Delta F$ & $\beta$ & $t$ \\
\hline Step 1 Model Summary & .02 & .03 & 2.45 & & & .01 & .02 & 1.48 & & \\
\hline Child Age & & & & .17 & 1.56 & & & & -.14 & -1.22 \\
\hline Step 2 Model Summary & .02 & .06 & 1.58 & & & .06 & .10 & 2.84 & & \\
\hline Ethnicity & & & & -.13 & -1.16 & & & & .22 & 1.91 \\
\hline Child Coaching & & & & -.15 & -1.27 & & & & .27 & 2.29 \\
\hline Step 3 Model Summary & .04 & .09 & 1.90 & & & .10 & .14 & 3.13 & & \\
\hline Ethnicity $\times$ Child Coaching & & & & .27 & 1.65 & & & & -.30 & -1.93 \\
\hline
\end{tabular}

Note. Child Coaching is centered.

${ }^{a}$ Ethnicity dummy coded $(1=$ Indian, $0=$ American $)$. 
Table 7

Summary of Regression Analyses Testing for Ethnicity as a Moderator of the Relationship between Regulation and CBCL Externalizing.

\begin{tabular}{lccccc}
\hline & \multicolumn{5}{c}{ CBCL Externalizing } \\
\cline { 2 - 6 } & $R^{2}$ & $\Delta R^{2}$ & $\Delta F$ & $\beta$ & $t$ \\
\hline Step 1 Model Summary & .02 & .03 & 2.45 & .17 & 1.56 \\
$\quad$ Child Age & & & & & \\
$\quad$ Step 2 Model Summary & .04 & .07 & 2.06 & -.13 & -1.14 \\
$\quad$ Ethnicity & & & & -.19 & -1.73 \\
$\quad \begin{array}{l}\text { Regulation } \\
\text { Step 3 Model Summary }\end{array}$ & .10 & .14 & $3.19^{*}$ & & -.47 \\
$\quad$ Ethnicity $\times$ Regulation & & & & $-2.49^{*}$ \\
\hline
\end{tabular}

Note. Regulation is centered.

$* p<.05$.

${ }^{a}$ Ethnicity dummy coded $(1=$ Indian, $0=$ American $)$. 
Figure 1: Interaction of Child Acceptance and Ethnicity (Indian or White American) in predicting CBCL Externalizing scores.

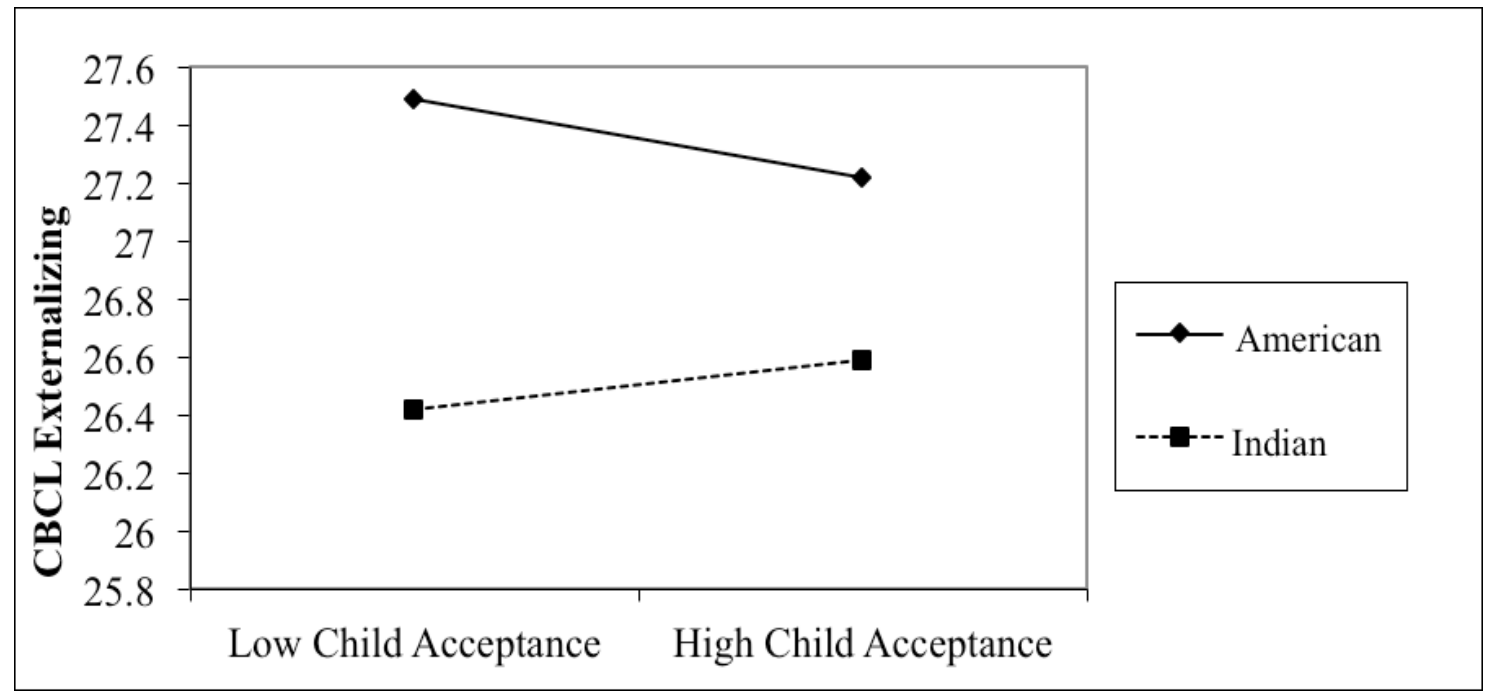


Figure 2: Interaction of Child Acceptance and Parent-oriented Interdependence (POISCS) Mean in predicting CBCL Internalizing scores.

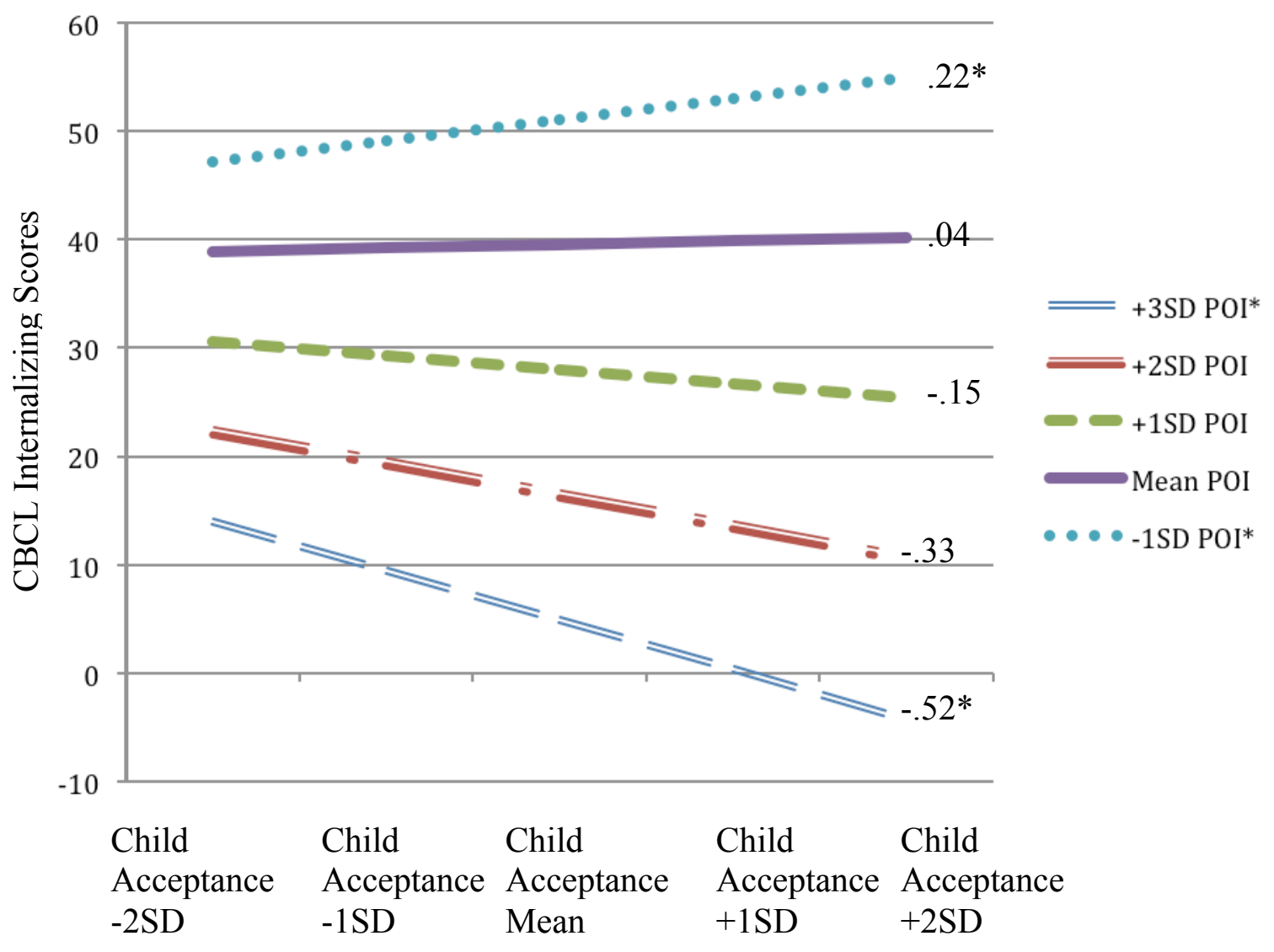


Figure 3: Interaction of Child Coaching and Ethnicity (Indian or White American) in predicting CBCL Externalizing scores.

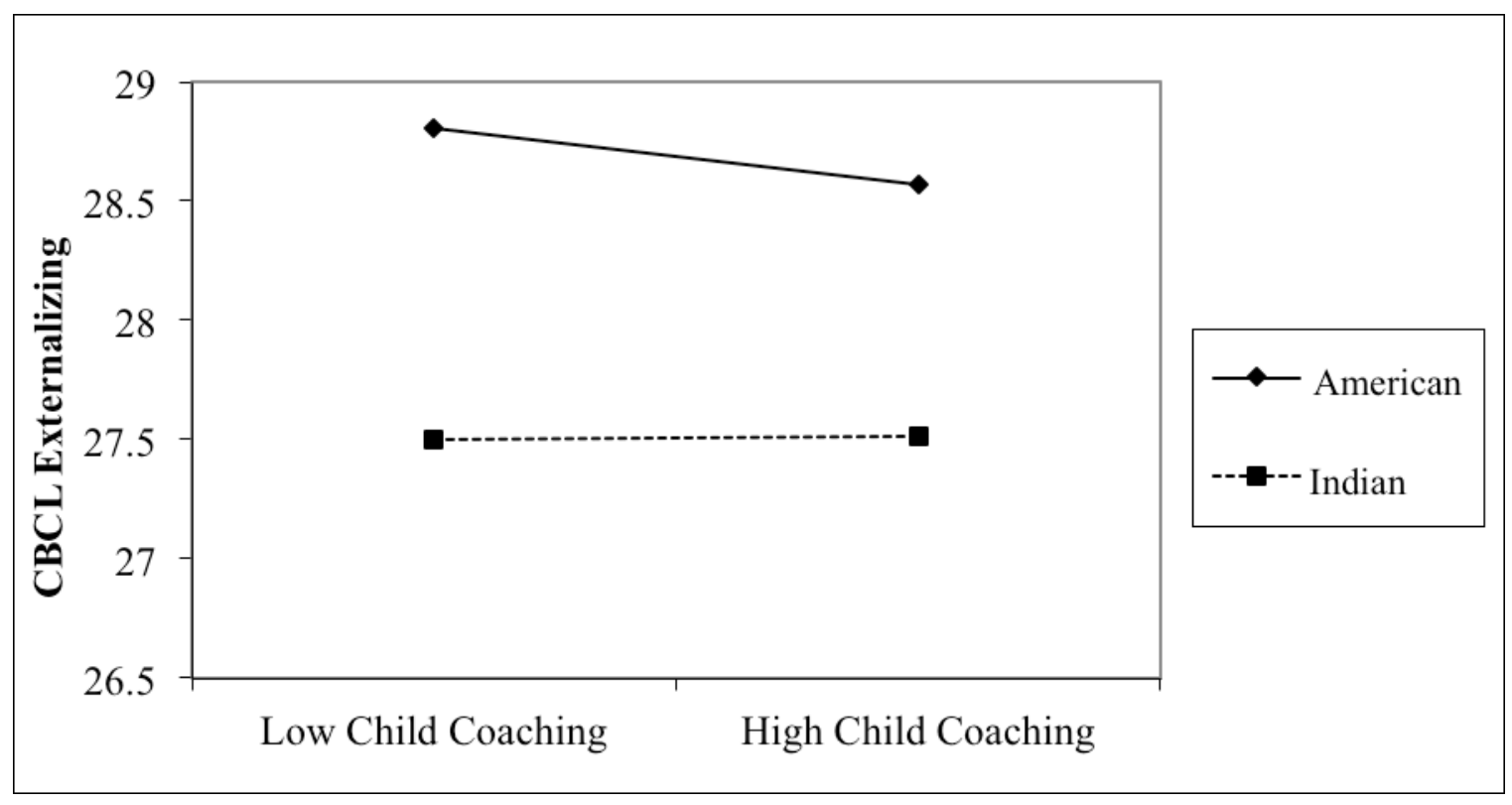


Figure 4: Interaction of Child Coaching and Ethnicity (Indian or White American) in predicting Mother-reported Home and Community Social Behavior Scales (HCSBS).

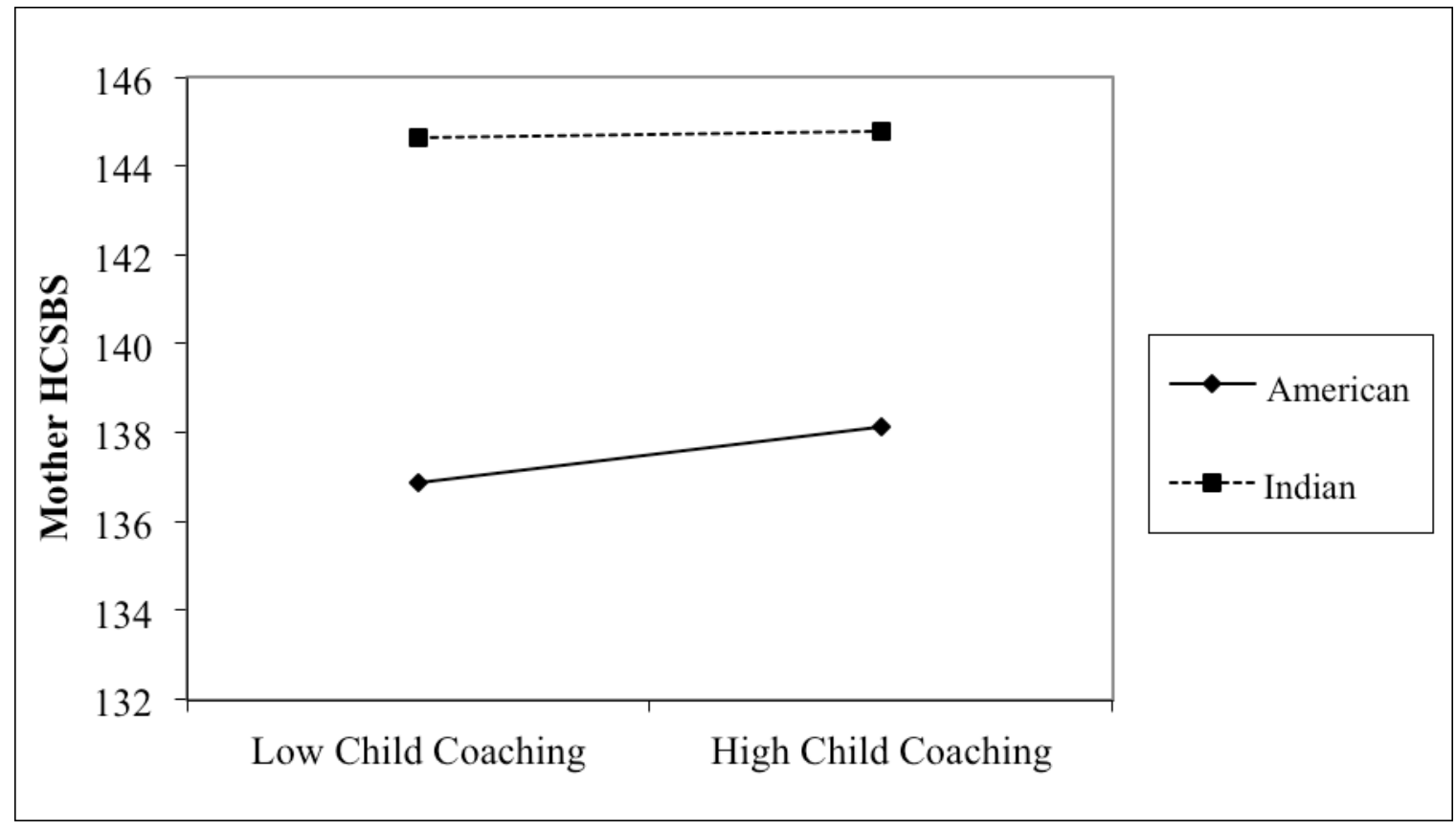


Figure 5: Interaction of Parent Regulation and Ethnicity (Indian or White American) in predicting CBCL Externalizing scores.

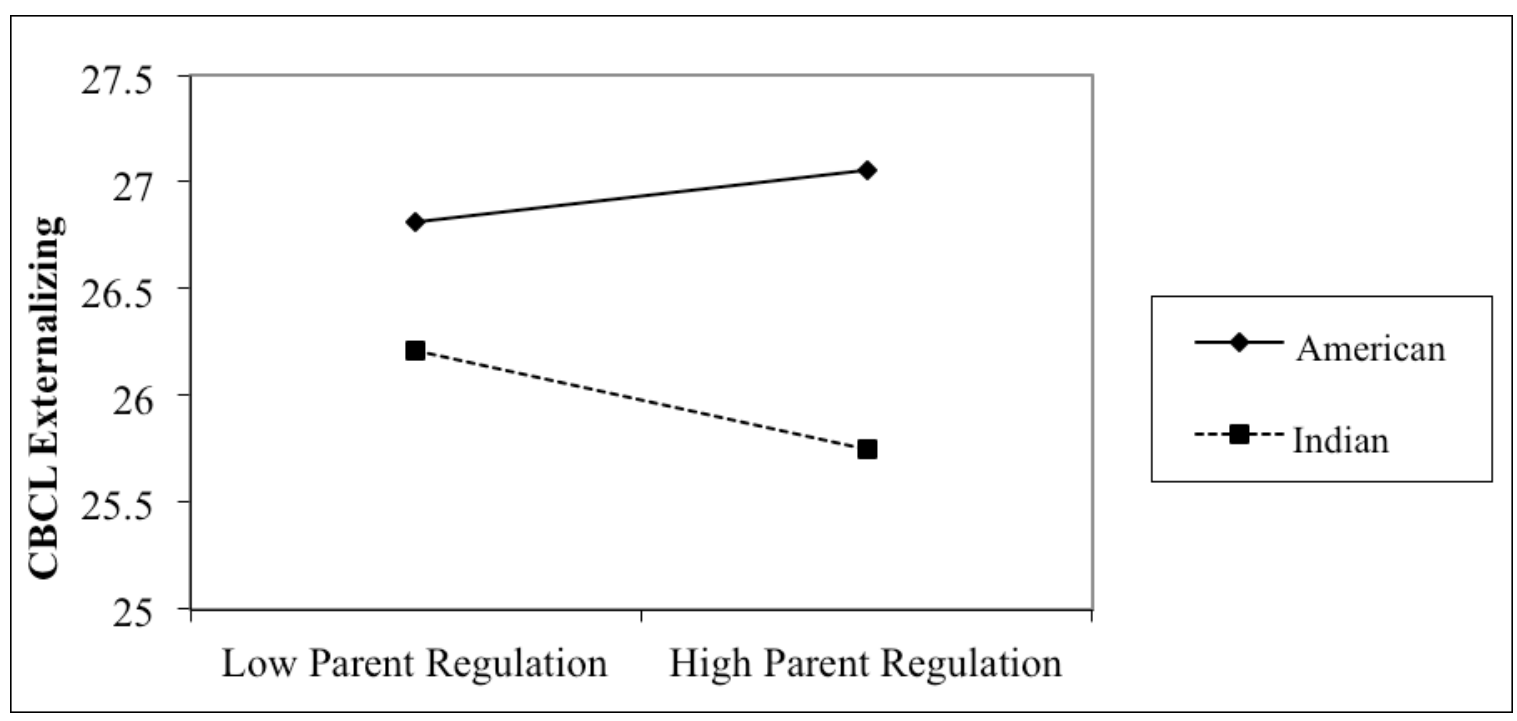

